\title{
La numismática y Melilla
}

Sería pretencioso por mi parte tratar de elaborar una historia de la ciudad de Melilla en la antigüedad, con el solo ingrediente y soporte de las monedas. No lo descartaría en vistas a un futuro no muy lejano. Es evidente que en las últimas décadas la NUMISMATICA como ciencia auxiliar de la historia, se ha revelado imprescindible en la aclaración de muchos hechos históricos. (La historia del imperio cartaginés, a falta de reliquias monumentales, han sido las monedas, como veremos en nuestro caso, el soporte para su reconstrucción histórica).

Las crónicas escritas y relatos de la antigüedad cuya lectura ha requerido muchos años de estudio y dedicación con las consiguientes controversias de los expertos, han aportado un ingente cúmulo de datos y hechos que constituyen las fuentes literarias, donde beben los investigadores de los pueblos antiguos. Quedan todavía por desvelar escrituras antiguas como el ibérico y el tartésico en nuestra península.

Pero al no aflorar nuevos documentos literarios la investigación se pierde en discusiones bizantinas y alambicadas en la interpretación de 
las fuentes conocidas, dando la sensación de que se encuentran muy exprimidas.

La Historia en las últimas décadas busca una revitalización y ampliación de sus conocimientos en la arqueología, ciencia ésta que permite corroborar o desmentir los datos aportados por las fuentes literarias e incluso ampliarlos.

La arqueología junto a las ciencias afines, la epigrafía y la numismática, cobran nueva relevancia como valores probatorios, sobre todo la última, gracias a los múltiples "tesoros" (así han dado en llamarle) de monedas que afloran como fruto, no sólo de las excavaciones arqueológicas, sino sobretodo a la utilización de las nuevas técnicas que aportan los detectores de metales. A este respecto tenemos que deplorar, una vez más, las expoliaciones denunciadas por los verdaderos investigadores y profesionales de la historia, quienes dan la voz de alarma ante hechos que califican como auténticas depredaciones fraudulentas, producidas por los artilugios ya indicados. Los detectores de monedas incontrolados, afloran innumerables lotes de monedas que en su mayoría pasan a manos de particulares, hurtánolas a la investigación de los expertos, perdiéndose uno de los datos más importantes: el lugar donde tuvo lugar el hallazgo.

Hagamos en este prólogo una aclaración sobre las limitaciones que las monedas ofrecen a la investigación histórica. En primer lugar el tiempo: su acuñación no se retrotrae más allá del siglo VII, límite cronológico para poder ser utilizadas como documento histórico. Tengamos en cuenta su localización en el espacio griego. Fueron los griegos los primeros que acuñaron monedas y desde Grecia el fenómeno se fue difundiendo a lo largo y ancho del MARE NOSTRUM. Y por último, en la antigüedad solamente las acuñaban los poderes públicos e instituciones de los pueblos de economía desarrollada y las utilizaban como instrumentos de cambio en sus transaciones.A la limitación del tiempo se añade que muchas monedas son anepígrafas y las que llevan leyenda, ésta acostumbra a ser escueta y poco elocuente. A pesar de todo el testimonio que aportan, es verídico al carecer de intencionalidad partidista. 
Las fuentes literarias históricas referentes a RLSADDIR, la actual Melilla, son abundantes, (no tanto como quisiéramos), y envidiamos las que tienen otras ciudades hermanas como CEUTA. En cuanto a las fuentes arqueológicas son deficientes, según Jesús M. Sáez Cazorla (TRAPANA n" 2, 1988, pág. 20) para quien "el método arqueológico no ha sido lo suficientemente aplicado".

Por ello son las fuentes numismáticas las que cobran especial relieve e importancia en el caso melillense, dada la ubicación de la ciudad en el ámbito mediterráneo, lugar de máxima difusión de las monedas antiguas.

En Ceuta el NUMARIO es tan rico, tanto el recogido en colecciones particulares como el aparecido en múltiples publicaciones sobre monedas ceutís que le ha permitido a mi amigo Carlos Posac Mon la publicación de un libro "LA HISTORIA DE CEUTA" a través de la NUMISMATICA (CELTA 1989) donde puede ir recogiendo a lo largo de los siglos las muestras monetarias que sirven de apoyo y corroboran los testimonios de las fuentes literarias a la vez que sirven de ilustración a la magnífica publicación.

En Melilla no tenemos material suficiente. Presumimos que los hallazgos de monedas en la ciudad y sus proximidades han tenido que ser numerosos pero han pasado, como siempre, a manos particulares y no han sido registrados en publicaciones, salvando algún caso esporádico como más tarde apuntaremos.

El presente trabajo sobre Numismática melillense intenta ser algo así como la primera piedra de un edificio a construir, un insignificante primer ladrillo, al que seguirán otros, no teniendo la menor duda de que ante la escasez de textos antiguos y a falta de unas excavaciones con metodología moderna, la aportación numismática puede contribuir decisivamente al esclarecimiento del devenir histórico de la ciudad de Melilla en la antigüedad.

Finalmente soy consciente de que el presente trabajo peca de subjetivo en la selección de las monedas, al no admitir como documentos todas las que se dicen haber sido halladas en el entorno de la ciudad. Solamente acepto y me apoyo en aquellas monedas que me ofrecen absoluta fiabili- 
dad y garantías de que su hallazgo ha tenido lugar en la ciudad y, si bien no han sido fruto de una excavación arqueológica, las circunstancias del hallazgo no generan dudas.

Al ser escasos los hallazgos, no tanto cuantitativamente, pero sí en cuanto a la cronología, solamente los podré aplicar a momentos y espacios temporales puntuales del ámbito fenicio, cartaginés y romano y dentro de éste analizaremos la repercusión del hallazgo de una moneda judía. La historia de la Melilla antigua se verá enriquecida.

En la actualidad no parecen darse voces discordantes sino que existe unanimidad por parte de los historiadores en identificar la antigua RUSADDIR, de nombre fenicio y lógicamente fundada por ellos, más tarde. romana,con la MALILA musulmana (en las fuentes aparece tal nombre en el siglo X) y la actual Melilla española.

Desgraciadamente nos consta la desaparición de las fuentes fenicias y cartaginesas que nos hablaban de su cultura, civilización y costumbres y por ende de la fundación de sus ciudades entre las que se encontraría lógicamente RUSADDIR.

Tanto el célebre naturalista romano Cayo Plinio (23-79. p. G.) como el "ITINERARIUM ANTONINI" atribuido al emperador CARACALLA (198-217 p.C) y recientemente a la época de Diocleciano, nos certifican la existencia de una RUSADIR romana que ostentó títulos como "OPPIDLM et PORTUS" e incluso "COLONIA".

También anotaremos que en cuanto a la "RUSADELRON" de CLAUDIO PTOLOMEO y la RLSCADA de Pomponio MELA, los historiadores no encuentran inconvenientes en identificarlas con RLSADDIR.

Queda sin embargo entre brumas la existencia e identificación con Melilla de una RUSADIR fenicia, constatada y documentada por fuentes fidedignas. Y la duda surge, no sólo por las contradicciones o más bien equivocaciones en que incurren las citadas fuentes greco-romanas al tratar 
de situar en sus coordenadas la ciudad de RUSADIR, son sin duda las deficientes excavaciones realizadas, y las que faltan por realizar las que no terminan de despejar las sombras existentes.

Son encomiables los esfuerzos de ENRIQUE GONZALVEZ GRAVIOTO (LA CIUDAD ANTIGUA DE RUSADDIR-MELILLA 1991) por demostrar la existencia de una RLSADIR FENICIA con argumentos, diríamos geoestratégicos, económicos e incluso acudiendo a otros posibles nombres que pudo tener la ciudad como AKROS, METAGONILM, etc. Yo mismo en sendos artículos en la Revista TRAPANA (n" 2 "MELILLA FACTORIA GRECA" 1988 pág. $13 \mathrm{y} \mathrm{n}^{\circ} 5$ "Península de TRES FORGAS en la ANTIGÜEDAD" 1991 pág. 37) abundaba en la misma línea de investigación.

Tenemos no obstante que confesar que las excavaciones realizadas por Rafael de Castro en el cerro de San Lorenzo nos certifican la existencia de una ciudad mauritana con influencias romanas cuya cronología no se retrotrae más allá del siglo III.

Y la esperanza de nuevos alumbramientos en futuras excavaciones parecen esfumarse, pues según el arqueólogo Dr. FERNANDO LOPEZ PARDO, solamente dos excavaciones al Oriente de Melilla han dado resultados positivos. Lo cual pone en entredicho los atlas de arqueología que presuponían establecimientos fenicios en nuestro litoral mediterráneo. La tesis del citado profesor de la Complutense en el sentido de que los fenicios fundaran una especie de metrópolis como UTICA, GADIR Y LIXUS desde donde se irradiaran a otros puntos de la costa,en el que su estacionamiento no fuera estable, parece cobrar fuerza.

Y llegados a este punto es la NUMISMATICA, la que nos ofrece argumentos de la existencia en el siglo I a. C. de una ciudad fundada por los fenicos con el nombre de RUSADDIR. Más adelante confirmaremos la presencia de cartagineses en la ciudad en el siglo III a. C.

Las monedas que portan el nombre de RUSADIR al parecer son un número muy reducido, solamente cuatro. $\mathrm{Y}$ de ellas, sólo una lleva la leyenda completa capaz de poderse leer claramente RUSADIR.

Pertenecen según noticias recogidas en publicaciones (JEAN MAZARD...) a dos tipos de acuñaciones diferentes, cuya descripción es la siguiente: 
PRIMER TIPO: ANVERSO, según MAZARD cabeza imberbe a izq. El jesuita español P. FIDEL FITA que la vio, matiza lo de la cabeza imberbe "cuyo tocado es el pellejo y orejas de un elefante".

REVERSO: según MAZARD, hay una abeja entre dos espigas y debajo la leyenda: $99 \$ 9 q$ (compuesta de cinco letras RESH, DALETH, ALEPH, SIN Y RESH que leidas de derecha a izquierda RuSADiR, intercalando dos vocales, la u y la i). El P.FITA corrobora lo dicho por MAZARD (F. FITA. "MELILLA PUNICA Y ROMANA". Bol. de la Real Academia de la Historia 1994, pág. 67).

Esta moneda, la más perfecta de las que se conservan y única, según MAZARD, acuñada en bronce, $22 \mathrm{~mm}$. de diámetro y 9,6 gramos de peso se conserva en el gabinete real de Copenhague (M. Supt. 215 a CH 141) de la cual se nos envió una fotografía el año 1990 después de proporcionarles los datos que el GABINETE REAL desconocía, y tenemos conocimiento que últimamente se ha enviado un vaciado a la organización $\mathrm{V}$ GENTENARIO DE MELILLA. El citado vaciado en dicha fecha nos fue prometido a ANUME, con incumplimiento de la promesa.

SEGUNDO TIPO de moneda cuya descripción es la que sigue:

ANVERSO: cabeza imberbe a izq. gráfila de puntos (MAZARD). P. QUINTERO la describe a su vez: cabeza de perfil a la izq. y gráfila de puntos (arte muy arcaico), reitera la descripción "abeja entre los espigas y debajo tres signos púnicos equivalentes a las letras RSA: gráfila de puntos. Es moneda por tanto atribuida a Melilla con su nombre fenicio Russadir".

Según MAZARD es de bronce y tiene $24 \mathrm{~mm}$. de diámetro $(2 \mathrm{~mm}$. más que el tipo primero) y pesa 11,2 gramos (1,6 gramos de diferencia con el primer tipo).

Este tipo no es único. Existen actualmente al menos dos monedas en espera de nuevos hallazgos: una en el Gabinete Real de Copenhague y otra en el Museo de Tetuán (Marruecos) según MAZARD.

Desconocemos si las dos son exactamente iguales en el diámetro y en el peso. Mazard nos informa de la duda que tuvo Müller en atribuir esta mone- 
da a Rusaddir, despejada por CHARRIER teniendo en cuenta la leyenda y el tipo. MAZARD añade que "el descubrimiento de otros ejemplares en las excavaciones de TAMUDA (Tetuán) suministra un argumento complementario".

Para este autor las dos monedas y y otras, que según él se han encontrado en las excavaciones, pertenecen al mismo tipo. Sin embargo P. QLINTERO (Rev, Mauritania 193 Diciembre 1943) resalta las diferencias entre las dos monedas. La cabeza de la moneda de Tetuán pertenece al dios BAAL, sinónimo de Saturno romano y la abeja sería la representación de ASTARTE. Por otra parte ésta se encuentra entre dos espigas y no entre un racimo de uvas y una espiga como afirma MAZARD.

Una cuarta moneda que según F. MATEL y LLOPIS se encuentra en los fondos del Museo del Instituto de Valencia de Don Juan en Madrid, a pesar de mis gestiones con la dirección del centro y la encargada del departamento numismático, se desconoce su existencia y hoy por hoy está desaparecida lamentablemente, si bien esperamos se reencuentre.

\section{APORTACIONFS IISTORIC:AS}

Dichas monedas, en primer lugar, reafirman la importancia de RLSADIR como emporio económico en su época ya que eran muy pocas las ciudades que acuñaban monedas, tanto en la Mauritania Tingitana como en la Cesariense, ya que tenían que gozar de autonomía propia: solamente se han encontrado monedas pertenecientes a trece ciudades: seis en la Cesariense: TEOSIM, IOL-CAE SAREA GUNUGL CAMARATA, TIMICI y SIGA y siete en la Tingitana: RUSADIR, TAMUDA, TINGIS, ZILI, LIXUS, SALA y TAMUSIDA.

Las monedas rusaditanas se han hallado,según noticias, dos en COL_CAESAREA (antigua capital de la Cesariense) y una en TAMUDA (TEUTAN), lo que parece indicarnos el comercio existente entre las tres ciudades pertenecientes a las dos Mauritanias: la oriental y la occidental.

El dato cronológico que aportan las monedas y que las sitúan en el siglo I a. C. nos permite deducir el papel importante económico que jugó nuestra ciudad en la época de JUBA II (25 a. C. -23 p. C.) "rey amigo y aliado de Roma" según el vulgo marioneta del emperador AUGUSTO. 
La Mauritania Tingitana gozó en esta época de gran prosperidad de la que sin duda se benefició la ciudad autónoma de RUSADIR. Si bien suponemos que la ciudad creció en importancia, una vez anexionada la Mauritania al Imperio Romano a la muerte de PTOLOMEO, asesinado por Calígula el año 40 p. C., hasta el punto de obtener el título de OPPIDUM y COLONIA, en tiempos del emperador CLAUDIO o lo más tarde de CARACALLA (212 p. C.). El explendor de la ciudad no pudo ser confirmado en las acuñaciones de monesdas, pues éstas fueron prohibidas en todas las provincias del Imperio durante el reinado de CALICULA (37-41 p.).

Otra cuestión importante que nos ofrecen dichas monedas es la IVTERPRETACION de sus EFIGIES y SIMBOLOS, la cual se presta a la elaboración de hipótesis de trabajo que a su vez generar controversias entre los investigadores. Las monedas antiguas acostumbraban a exculpir en sus anversos deidades nacionales y en época helenística aparecían las cabezas o bustos de sus reyes o jerarcas. Era una forma de autentificar el valor de la moneda.

En el primer tipo de las monedas de RUSADIR, la efigie imberbe y tocada con la piel y oreja de un elefante podría representar a un dios local que al igual que HERCULES portaba una clava, el nuestro cubría su cabeza con la piel de uno de aquellos paquidermos que habitaban los frondosos montes del RIF cercanos a Melilla y que Anibal utilizó en su marcha sobre Roma. La cabeza del segundo tipo, según QUINTERO, representa al BAAL cartaginés, el Saturno romano.

Las dos últimas parecen referirse a los recursos que tenía la ciudad: el trigo o cebada que representan las espigas, no parece que fuera muy abundante, dado el espacio geográfico circundante a la ciudad que los antiguos represenbtan como un desierto; pero que bien pudo darse, dada la abundancia de agua (actualmente TRARA y YASINE) o la importación de trigo en ánforas que han aparecido en aguas del entorno melillense. La uva tampoco la podemos descartar, pues pertenece a un producto de la trilogía mediterránea.

Existe para mí un dato que lo juzgo significativo y es el símbolo de la ABEJA que portan las cuatro leyendas, fundamentándome en el idéntico símbolo, si bien más artístico, que llevan diferentes monedas griegas con 
una leyenda en griego que puede leerse $\mu \varepsilon \lambda y$ (MELY-TOS en el dialecto ático significa $\mathrm{ABEJA}$ ) asociada al símbolo de la susodicha abeja, me permite deducir la posibilidad de que el nombre de nuestra ciudad, en árabe MALILA o MELILLA provenga del griego MELITA que recalcamos en dialecto jónico significa abeja, productora de la miel. Esta hipótesis de trabajo no puede considerarse absurda. Los linguiistas y la filología comparada podrían ofrecer argumentos en favor o en contra y sobretodo las excavaciones arqueológicas a realizar en la ciudad, tendrían la última palabra.

El argumento etnológico del entorno melillense como he apuntado en diversos artículos, prueban, o bien la presencia griega en la región o la aportación de elementos culturales griegos por otros pueblos que bien podrían ser los fenicios.

Mayores dificultades presenta la interpretación de la abeja. Quintero le da un significado religioso: representación de la diosa ASTARTE (la TANIT cartaginesa) cuyo culto pudiera tener lugar en el promontorio de Melilla la Vieja. MAZARD siguiendo a MÜLLER prefiere darle un significado económico, pues según estos autores, la ciudad de Melilla toma su nombre de los recursos importantes procedentes de la apicultura. Según ellos, Melilla viene de MIEL. Quizá estos autores leyeron a Marmol de Carvajal y León el Africano, quienes abundan en la riqueza de miel que produce la apicultura de la ciudad o al significado que algunos autores, como Juan Antonio de Estrada "POBLACION GENERAL DE ESPAÑA, 1847", atribuyen el nombre árabe de MALILA, etimológicamente a MELLOSA por su miel.

El tema de la abeja da rienda a la especulación. En un trabajo publicado en TRAPANA n 2 1988, pág. 13, al cual me remito, decía que sería una aportación más al origen y etimología del nombre de Melilla tan discutido e ignorado.

Antes de concluir el tema de las monedas de Copenhague y hacer historia de cómo han llegado a nuestro conocimiento, añadiremos que desconocemos si el hallazgo de estas monedas que tuvo lugar en CHERCHEI, (Argelia) antigua IOL-CESAREA según MÜLLER, fue fruto de una excavación o el hallazgo fue casual. 
No sabemos si las monedas existían cuando el rey de Dinamarca Cristian VII decidió en 1843 publicar una obra sobre las "Medallas de la Antigua Africa" teniendo en cuenta las series o colecciones excepcionalmente ricas que se conservaban en el GABINETE REAL. Al fallecer los dos sabios encargados de la obra en 1857, fue encargado de proseguirla LUWIG MÜLLER director de dicho Gabinete Real. En un tiempo relativamente corto publicó los tres volúmenes. El primer tomo apareció en 1860 y el último dedicado a la Mauritania en 1862. Más tarde en 1874 LUDWIG MÜLLER publica un suplemento titulado "NUMISMATIQUE de LANGIE.NE AFRIQUE" COPENHAGLE 1874. En él corrige las incorreciones de su obra anterior y añade datos nuevos.

Esta obra fue la que llegó al conocimiento del jesuita P. F. FITA y en una publicación sobre "Melilla púnica y romana" en el boletín de la Real Academia de la Historia el año 1914, dio a conocer a la comunidad científica la existencia de una de las monedas mejor conservadas con la leyenda RLSADIR. Esa publicación llegó a ser conocida por Rafael Fernández de Castro que nos la da a conocer puntualmente con el dato importante para Melilla de la existencia de la citada monedad en MELILLA PREHISPANICA. (MADRID 1945), así como la que encontró P. QUINTERO en las excavaciones de TAMUDA. Esta anédota revela la lentitud en que llegó al conocimiento de los melillenses el hallazgo de una moneda tan relevante para la historia de la ciudad.

\section{IAA MELILLA PUIYICA}

El apelativo de púnica o cartaginesa lo tiene de sobra merecido nuestra ciudad, después de la lluvia de miles y miles de monedas que cayeron en tromba desde lo alto del tubo colocado al otro lado del dique o espigón del puerto. Dicho tubo procedía de la plataforma y estaba conectado con el que practicaba la draga succionando los fondos marinos del puerto. Se revelaron más tarde que la mayor parte eran púnicas o cartaginesas. 
Este espectáculo de las monedas caidas, al decir de alguros "del cielo", quedará imborrable en la historia de la ciudad. Es lamentable constatar que sólo un porcentaje pequeño de las que fluían del tubo han podido ser rescatadas y conservadas bien en manos de particulares o en el Museo de la Ciudad. La mayor parte de las monedas que fueron succionadas en la draga del puerto volvieron inexplicablemente al mar fuera del puerto y se perdieron entre las piedras de la escollera, en su lado exterior unas y las más, inevitablemente fueron a parar al fondo del mar.

Ocurrió este hecho el año 1981 en cuya fecha denunciamos la incuria y dejadez de nuestras autoridades, en especial las culturales, ante un acontecimiento de tal magnitud al no adoptar las medidas cautelares para evitar lo anteriormente reseñado. Y más cuando había constancia de que en una draga anterior del puerto efectuada el año 1953, habían sido rescatadas unas docenas de estas mismas monedas. Incluso tengo noticias que un buzo alemán consiguió algunas en una prospección que realizó en los cimientos del cargadero de mineral de la antigua Compañía del Rif.

Evidentemente el tema de las monedas no despertaba la sensibilidad que hoy despierta en el mundo cultural y científico, al desconocer el valor documental que tienen como prueba histórica a falta de otra documentación. Nos queda constatar, refiriéndonos a otro tipo de monedas, que son docenas o centenares las monedas afloradas y encontradas de forma fortuita en Melilla, que han vuelto a desaparecer en los bolsillos o rincones de las casas sin quedar constancia de su descubrimiento y poder servir de documento histórico. Desde aquí un llamamiento para que no se repitan estas incurias.

Volviendo a las monedas púnicas de la draga que al ser miles oscurecen las pocas que se han encontrado en la tierra firme, constatamos que han sido objeto solamente de dos estudios. El que realizamos juntamente el amigo Don Salvador FONTE.NLA Ballesta al poco de su aparición y que se publicó en la Revista n” 132.000 de Madrid (Enero 1987) y el último aparecido en la Revista NUMISMA 232 Año XLIII de Madrid / Enero-junio 1993) y firmado por la conservadora numismática del Museo Arqueológico Nacional de Madrid, Carmen Alfaro Asins. 
Nos remitimos a estas dos únicas publicaciones, la primera Revista 2.000 1987, dirigida al mundo del coleccionismo (la Revista es de la Asociación de Numismáticos profesionales) y la segunda NUMISMA 1993, da a conocer el descubrimiento de las monedas melillenses al mundo científico.

Matizaremos que el estudio de las monedas, tanto el nuestro como el efectuado por Carmen Alfaro Asins, lo ha sido sobre un lote que no sobrepasa las 700 monedas. Restan las más de 2.000 del Museo de la Ciudad y otros miles en posesión de particulares que convendría fueran examinadas y estudiadas como lo han sido las anteriores, si bien estamos seguros no cambiarían las conclusiones a las que se han llegado en la última citada publicación. Estamos de acuero con el documentado artículo de Carmen Alfaro, en el que se llega a la conclusión de que más del 90\% de las monedas tanto las de ELECTRON (oro $30 \%$ y plata) corresponden al período cronológico comprendido entre 221 y 202 a. C. correspondientes a los años que transcurren entre los "prolegómenos y durante la Segunda Guerra Púnica".

En el lote de las 700 monedas estudiadas, Carmen Alfaro ha encontrado algunas "residuales" pertenecientes a períodos cronológicos anteriores:

$1^{\circ}$. Una sola de cobre casi puro ha conseguido datarla a mediados del siglo IV a. C.

$2^{\mathrm{D}}$. Otras cuatro de bronce que se diferencian del resto en el protome o cabeza de caballo que figura en el reverso en lugar del caballo entero. Pudieron ser acuñadas en torno al 300-264 años a. C.

$3^{\prime \prime}$. Seis monedas de vellón en cuyo reverso una palmera atraviesa el campo verticalmente ocupado por el caballo mirando a la derecha o en la grupa se ve una estrella de siete u ocho puntas o también un URAEUS egipcio. Se trata de DISHEKELS y SHEKELS de vellón datables entre los años 264-221 a. C.

El pequeño error cronológico que cometimos el Sr. Fontenla y yo en la primera publicación es achacable a las dificultades de clasificación que ofrece este tipo de monedas, ya que presentan una uniformidad de tipos invariable a lo largo de su vida. En el anverso la cabeza de TANIT O PER- 
SEFONE siempre a izquierda en pequeñas variantes (ver art. en $\mathrm{N}^{0} 2.000$ n” 14, pág. 17), se nos presenta en unos casos más joven que en otros. Las monedas cartagniesas peninsulares desaparece el rostro de mujer reemplazado por otro varonil que presumiblemente representa a Aníbal. Tanto las monedas africanas como las peninsulares son anepígrafas, sin leyenda en el anverso.

En el reverso aparece invariablemente un caballo al paso que mira siempre a derecha con la cabeza hacia adelante (de perfil) o hacia atrás (retrospicente).

Los símbolos son variados: palmeras o caduceos que atraviesan todo el campo partiendo en dos la figura equina; estrellas de seis, siete u ocho puntas, a veces el ureus egipcio en el espacio superior entre la cabeza y la grupa del caballo y por último una palmeta que surge pegada a la grupa. Letras o signos púnicos colocados bien en la parte delantera o entre las patas tales como ALEPH, SIM, GUIMEL, BETH, DALETH, LAMED, etc.

Se da la circunstancia de que "monedas con idénticos tipos (Carmen Alfaro o.p.e. 11) pueden haber sido acuñadas en diferentes épocas y lugares. Tan sólo pequeñas diferencias a veces difíciles de precisar pueden ayudar a situar las emisiones en su contexto".

Subrayamos las dificultades que presenta la clasificación cronológica de este tipo de monedas, que como hemos reseñado antes, nos indujeron, al parecer, a equivocarnos. En el esfuerzo por resumir los tipos que pueden ofrecer variaciones en el lote de monedas $(90 \%$ del total) comprendidas cronológicamente entre los años 221 al 202 a. C.. nos fijaremos solamente en el reverso, pues las variaciones del anverso, lo hemos apuntado, son mínimas: pendientes de uno o tres colgantes y madurez o juventud de la diosa cartaginesa.

En el reverso de las monedas de electrón (oro y plata) sólo se aprecian dos tipos según la posición del caballo: parado o al paso.

En las monedas de cobre se pueden apreciar variantes, teniendo en cuenta la posición del caballo al paso o parado, mirando hacia adelante o hacia atrás (retrospicente) y el acompañamiento de símbolos tales como soles o estrellas, palmetas y caduceos, por lo que pueden establecerse hasta seis tipos: 
$1^{\circ}$. Caballo al paso retrospicente.

$2^{\circ}$. Caballo parado retrospicente.

$3^{\circ}$. Caballo parado retrospicente y detrás estrella de seis, siete, ocho o nueve puntas.

$4^{\circ}$. Caballo parado retrospicente y detrás palmeta.

$5^{\circ}$. Caballo al paso no retrospicente con caduceo.

$6^{\text {o }}$. Caballo parado no retrospicente con estrella de ocho puntas.

Según Carmen Alfaro se echa en falta un $7^{\circ}$ tipo: caballo retrospicente con caduceo que Jemkins atribuye a la Segunda Guerra Púnica, presente según él, en un tesoro de BUJIA en la costa marroquí, no lejos de Melilla.

Los caballos están en posición todos hacia la derecha; cuatro tipos con la cabeza hacia atrás y solamente dos tipos miran hacia delante de perfil.

Según la citada autora, el lote melillense está representado en otro lote más pequeño formado por 56 monedas, encontrado en Torre de Doña Blanca (Puerto de Santa María, Cádiz), con motivo de la campaña de excavación de 1986 en una de las habitaciones de la zona denominada del "espigón" (o.p.c. pág. 28). Este hallazgo nos lleva inevitablemente a la discusión sobre el lugar de acuñación de ambos lotes que según Carmen Alfaro pertenecen a los mismos tipos e incluso en su composición presentan el mismo alto porcentaje de plomo en algunos casos cercano al $90 \%$.

Tanto el lote melillense, ubicado en la costa africana, como el gaditano localizado en el litoral peninsular andaluz, para la citada autora tienen una misma cecca o lo que es lo mismo. su acuñación ha sido efectuada en Cartago de Africa arguyendo el alto porcentaje de plomo que tiene tanto el lote melillense como el gaditano. Este mineral ha sido explotado en la antigüedad en la zona llamada Yabat Rusas o "montaña de plomo" localizada en las afueras de Túnez. Las monedas cartaginesas acunadas en España no tienen aleación de plomo sino de cobre.

Nosotros discrepamos de Carmen Alfaro en el lugar de acuñación del lote melillense. Admitimos que las monedas de Electrón 3/8 de Shekel (según Jenkis y Lewis $3 / 4$ de dracma fenicia o tres tribemiobolos) pueden tener una acuñación foránea, bien en Italia (Robisnson) o en Cartago (Jen- 
kins) en Hispania (Villaronga). Estimamos que la acuñación de estas monedas necesita excelentes toreutas y un taller ciudadano bien organizado inexistente a nuestro parecer, en la Rusadir púnica, si bien las materias primas; el oro procedente de Sudán y la plata de Cartagonova, podrían muy bien converger en Melilla.

Las monedas de bronce de alto porcentaje en plomo, llamadas también "de necesidad". SHEKELES y DISHEKELES pudieron muy bien acuñarse en Melilla en los que Villarronga Ilama "talleres militares móviles", donde los generales cartagineses acunaban con escasos recursos y medios en los que los artistas abridores de cuñas no pasaban de una categoría artesana muy mediocre". (L. Villarronga: "Monedas Hispano-Cartaginesas". 1973, pág. 110).

GUADAN (MONEDA IBERICA 1980) atribuye a TALLERES INDETERMINADOS DE TIPO MILITAR la acuñación de monedas anepígrafas de cabeza femenina análogas a las nuestras. Como apuntábamos en la publicación N 2000, pág. 10, 1987, "los cospeles los obtuvieron en moldes por fusión para varias piezas tomando la forma de árbol.

Al separar los restos de los canales intermedios raramente han sido pulidos para disimularlos. El cospel restante es circular y con el perfil ligeramente tronco-cónico correspondiendo la cara menor con la parte inferior del molde.

La forma de acuñación nos lleva a pensar en un taller itinerante localizado en Melilla, donde un alto contingente de tropas como de todos es conocido, estaba acantonado en sus inmediaciones y lógicamente demandaba un alto numerario para sus transaciones cotidianas. El plomo con el que estan acuñadas nuestras monedas en un porcentaje que puede alcanzar el $90 \%$, lo tenían a escasamente a $20 \mathrm{kms}$. de Melilla en el yacimiento que conocemos con el nombre de "MONTE AFRA". Según Ginés Sanmartín, ingeniero técnico de minas (Rev. Trápana $n^{\circ}$ 2, 1988 "la Compañía del Norte Africano", pág. 17) concesionaria de los yacimientos mineros del "MONTE AFRA" al comienzo de sus actividades en 1908 se encontró en las antiguas escavaciones una pequeña figura de bronce clasificada como Astarté, diosa sirio-fenicia. 
Esta anécdota a falta de excavaciones que cerifiquen la explotación de esta mina en época cartanginesa (concretamente a finales del siglo III a. G.), nos sugiere la hipótesis de la acuñación local de las monedas extraídas del puerto de Melilla.

En cuanto a la aportación histórica que nos ofrecen estas monedas, estamos todos de acuerdo.

$1^{\prime \prime}$. Confirman las citas literarias del romano Tito-Livio y el griego Polibio del acontonamiento de tropas de los bárcidas en Melilla y su entorno, así como el trasvase recíproco de militares entre la costa peninsular y la africana. Las monedas parecen coincidir con el momento más álgido y crítico de la Segunda Guerra Púnica: cuando los cartagineses han perdido Cartagonova (209 a. C.) y están a punto de ser expulsados por los romanos de la Península al abandonar Gadir en el 206 a. C.

$2^{\circ}$. Los cartagineses imposibilitados de acuñar las monedas en la Península, recurrieron a a efectuarla en la ceca melillenses. Destaca el valor estratégico de Rusadir como enlace de Cartago en la Península y sus puertos Gadir, Malaca y en especial su capital Cartagonova. En época romana la importancia estratégica de Melilla-Rusadir, aumentaría. Las corrientes marinas, el puente natural entre el cabo de Tres Forcas, la isla de Alborán y Adra en Almería, que une dos continentes y Africa con la Península, confieren a Melilla el valor estratégico que la convierten en protagonista de acontecimientos históricos.

Queremos subrayar el dato ya apuntado, que la mayor parte del lote de monedas están sin estudiar, La importancia de dicho lote está pidiendo, exigiendo, la publicación del mayor número posible de monedas desperdigadas en colecciones particulares y sobre todo, las que están en el Museo Municipal de la ciudad.

MELILLA ROMANA: EPOCA CONSULAR (SEGLNDO SIGIO A. C.)

Después del esplendor del numario cartaginés aflorado casualmente en Melilla, el período romano no luce numismáticamente como el anterior. 
Los testimonios literarios nos hablan de una Rusadir que llegó a alcanzar el máximo título que podía aspirar una ciudad indígena mauritana: el de Colonia Civium Romanorum.

Las noticias sobre hallazgos de monedas romanas de forma fortuita en Melilla son constantes. Pero sus posesores o las han perdido o las ocultan. Sorprende que en las pocas excavaciones realizadas en la ciudad no ha quedado constancia de ninguna.

Las que se hallaban en el museo de la ciudad han sido robadas recientemente, si bien queda constancia de su existencia gracias a un catálogo que realizó de ellas el amigo Salvador Fontenla Ballesta (Trápana $\mathbf{n}^{\circ}$ 2, 1988, pág. 75). Figuran 2 ases de Caludio I y 1 sextercio de Trajano y Follis de Constancia I, Constantino III, Constancio II Majoriana y un centonial de Honorio.

Como no nos consta su procedencia de Melilla y según Fontela, algunas habían sido encontradas en Mérida, no podemos relacionarlas con nuestra ciudad. Nuestra suerte quiso que en agosto de 1983 se produjera el hallazgo, a mi juicio el más importante habido en la ciudad, después del cartaginés, aunque se tratara de una sola moneda: un as Uncial cuyo peso, dato importante, fue de 28,6 gramos.

El hallazgo se había producido cuando un jardinero municipal procedía a la plantación de un árbol, previa la excavación de una cava u hoyo en las cercanías del actual cementerio municipal. La moneda en cuestión goza, según mi apreciación, de todas las garantías al igual que si hubiera sido extraída en una excavación científica. Dado que en las proximidades del lugar se habían encontrado cerámicas de terra sigilata romana, e incluso se ha especulado con el hallazgo de monedas.

La descripción de el As Uncial es la siguiente: en el anverso puede distinguirse un Jano bifronte y en el reverso una proa de nave con la leyenda Roma en el exergo, debajo de la proa. Una marca o trazo vertical grueso, denota que se trata de un as uncial.
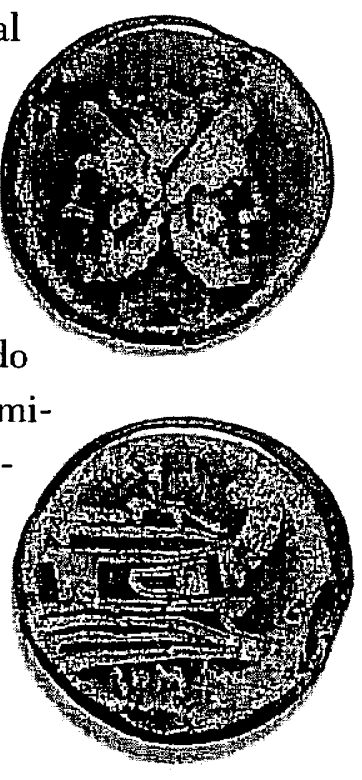

As Unicial ballado en Melilla. 
El material en el que está hecho es de bronce, aleación de cobre y estaño, pesando como ya hemos apuntado 28,6 gramos y un diámetro de 33 milímetros.

La historia de los ases unciales deriva del primer as grave o libral que Roma acuñó en el siglo IV a. C., cuyo peso era de 327 gramos, teniendo en el anverso curiosamente un Jano bifronte igual al nuestro.

El peso del "as grave" fue disminuyendo hasta alcanzar el año 221 a.C. 54,5 gramos de peso y tomó el nombre de "as sextantal". Pocos años más tarde, el 217 p. G., la ley Flaminia determina la creación de un nuevo as, bajando el peso a la mitad, 27,5 gramos y cuya denominación será "as uncial". Esta última devaluación tuvo como causa, la guerra contra los cartagineses.

Una de las publicaciones más importantes en orden a determinar y fijar la fecha de este tipo de monedas, los ases unciales, es de la de (M. Crawford "Roman Republican Coinage" Cambridge University, 1974), donde este autor inglés realiza un estudio en el que va siguiendo cronológicamente las sucesivas devaluaciones que han venido padeciendo los ases unciales hasta alcanzar los 28 gramos, el año 158 a.G, (ver gráfico), justamente el peso aproximado que tiene el hallado en Melilla al ser ligeramente superior situamos su acuñación poco antes de la citada fecha del 158 a.C.

En cuanto a la relevancia que pueda tener dicho hallazgo viene dada en función de ser el primero del que hay constancia de su aparición, no sólo en Melilla, sino en toda la zona del que fue Protectorado Español en Marruecos.

La información que aporta Enrique Gonzálvez (cit. op. pág. 199) del hallazgo de otros ases unciales en Marruecos, concretamente en Tamuda (Tetuán), la juzgó equivocada. Gonzálvez se apoya en la publicación del reconocido a investigador numismático D. Felipe Mateu y Llopis ("Monedas de Mauritania, Madrid 1949). Los datos de dicha obra, a mi juicio, no están correctamente tomados, puesto que en la página 35, citado por E. Gonzálvez, los susodichos ases, según Mateu y Llopis son inclasificables aportando el dato de su peso: 22 gramos. En el caso hipotético que se tratara de ases Unciales en ningún caso la cronología podría ser anterior al as uncial melillense dado el peso de los citados. 
$\mathrm{El}$ as uncial de Melilla nos lleva o induce a sugerir la presencia de tropas militares o comerciantes en la ciudad durante la mitad del siglo II a.C.

Los restos aflorados en las excavaciones archiconocidas del Cerro de San Lorenzo ponen de manifiesto la relativa importancia de la Melilla mauritana y romana de los siglos III y II a.C.

Las relaciones de nuestra ciudad con Malaka a mediados del siglo $2^{\circ}$ antes de nuestra era, han sido puestas de manifiesto por múltiples autores entre los que resaltamos a E. Gonzálvez. En dicho libro se lee textualmente: "sabemos que (Malaca) en la antigüedad mantuvo relaciones numerosas con esta costa (Melilla) de la que hay evidencia incluso en las fuentes literarias, máxime cuando Malaca parce que se especializó notablemente en el comercio con la zona mediterránea de la Mauritania". Cita a Rodríguez Oliva con dos testimonios epigráficos de los contactos entre Malaca y los territorios norteafricanos. Nosotros mismos abundábamos en el tema (Trápana $n^{\circ} 2$ 1988, pág. 14) y escribíamos de estas citas (Polibio Estrabón, etc.) puede deducirse la estraordinaria importancia que tenía el comercio de la región norteafricana en torno a Melilla con el emporio comercial de Málaga en los siglos anteriores a nuestra era...). Estos testimonios avalan la existencia de comerciantes en ambas ciudades.

Sin embargo, el tema de la presencia de tropas militares romanas en Melilla es más sugestivo. Nos consta que en Melilla se acantonaron tropas durante la Segunda Guerra Púnica.

En los años en torno al 158 no existía la provincia creada por Roma y la que más tarde se llamaría Mauritnaia Tingitana. El rey sumiso a Roma que dominaba esta región la Numidia, se llamaba Masinissa, quien heredó el reino de Gaia hacia el año 208 a.C.; inclinado a la causa romana vence a Syphax aliado de Cartago y se anexiona gran parte del reino masaesilio. La conquista de Cirta el año 203 a.C. marca los comienzos del engrandecimiento de Masinisa. CH.A. Julien (Histoire de L'Afrique du Nord (pág. $1041^{a} \mathrm{ed},+$.) ha escrito de él: "fue un gran "Agvellid" que amasa un pueblo con sus poderosas manos y hace de la Berberia un estado unificado e independiente. Si el jefe bereber se ve imposibilitado por el imperialismo 
romano de alcanzar el fin que casi tocaba con sus manos, al menos su tentativa pone de relieve sus cualidades excepcionales de soberano".

El engrandecimiento del reino númida se realiza a partir del año 193 en el que Masinisa ve como sus tierras aumentan con sucesivas conquistas en detrimento de Cartago ya que el tratado del año 201 priva a este reino de los medios de defensa. Firmado éste en Roma, consta que Scipión había reconocido a Cartago la posesión de los territorios situados al oeste de las "fosas fenicias" pero Masinisa estaba autorizado a reivindicar en el interior de estos límites, las tierras que habían pertenecido a sus ancestros. El rey númida, masylio utiliza esta cláusula que se revelara ser el verdadero origen de la Tercera Guerra Púnica. CH. Saumagne ha demostrado justamente que Masinisa utiliza los argumentos jurídicos los más eficaces, demostrando que Cartago retiene sus territorios por la fuerza ( $y$ no por derecho) ya que no posee ningún "Propius Ager" territorio propio y el origen mismo de la posesión por parte de Cartago es injusta. "Diriamos que Masinisa abre el el proceso al colonialismo" (Gabriel Camps "Bereberes" pág. 149 Hespérides 1980). Así el año 162 a.C. se anexiona (descoloniza) una gran parte de los "Emporia" y nueve años más tarde de los "Campi Magni".

Antes de la destrucción de Cartago (146 a.C.) el senado había convertido prácticamente el reino numida en un estado "protegido" confiado (según G. Camps op.cit.), más que entregado a MASIMISA pág. 158). "Este, según Camps, fue un gran rey y un hábil político que ha tenido siempre muy clara su condición de "rey cliente", condición que en su tiempo se calificaba de "amigo y aliado del pueblo romano". Invocando este título el cónsul Nobilior en situación crítica asediando a Numancia el año 153 a.C., solicita ayuda de Masinisa. En torno al año 150 a.C. fecha próxima a la acuñación de nuestro as uncial, Masinisa cuenta con 88 años de edad y sabemos que a esa edad montado a caballo conducía a los numidas en la batalla; en esta ocasión decidió no ir él en persona a Numancia, sino que prefirió enviar al cónsul Nobilior una ayuda de 300 jinetes, tropa famosa por su excelente adiestramiento y eficacia en el combate con arco sobre una montura y también envió diez elefantes. La llegada de estos refuerzos, en especial los elefantes, animó a Nobilior a atacar la ciudad. Los indígenas (arévacos y sege- 
danos) asustados al contemplar esos animales por primera vez, se replegaron. Pero una piedra lanzada desde las murallas hirió a uno de los animales que enloquecido se precipitó sobre los propios romanos. Aprovechándose del desconcierto, los indígenas arévacos produjeron otros 4.000 muertos en las fuerzas romanas (Cuadernos de Historia $16 n^{0} 26,1985$ ). Como vemos la ayuda de Masinisa no tuvo el éxito previsto.

Pero en nuestro interés podemos aventurar que dicho envío de los 300 jinetes y 10 elefantes a Numancia, ruta lógica era a través de la isla de Alborán a la Península ya que Melilla peretenecía en estos tiempos a la Numidia. Suponemos que Roma a través del cónsul Nobilior envió el suficiente numerario para contratar los antedichos efectivos militares en la región melillense.

Masinisa murió el año 148 a.C. dejando 44 hijos y encargando al tribuno Scipión Emiliano (siendo cónsul rindió Numancia el año 133 a.C.) amigo suyo que como albacea arreglara la sucesión. Gsell ha escrito "Masinisa termina su vida en la predicción de que los destinos de la Numidia dependían de los romanos. Esta larga cita de Masinisa se basa en la intuición aunque no hay certeza de que nuestro as uncial tuvo que ver con el famoso rey de Numidia.

MELILLA ROMANA: FPOCA IMPERIAT.

Tampoco de esta época contamos con numerario abundante como el de Ceuta. ("La historia de Ceuta a través de la numismática" de Carlos Posac Mon, Ceuta Nov. 1989).

No quiere decir que no se hayan producido los hallazgos con esta cronología, más bien en el caso de haberse producido no se han conservado y se ha perdido la memoria de su hallazgo. La historia de Melilla referida al alto imperio conserva los datos escuetos transmitidos por las fuentes romanas de Plinio y el Itinerarium Antonini, ya citados con reiteración.

Desearía resaltar la importancia de un hallazgo numismático ocurrido en 1995. Entre un lote de monedas antiguas encontradas en Melilla, casi todas ellas frustras, había una que al limpiarla me produjo una grata impresión. 
En otra ocasión no le hubiera dado importancia, pero se trataba de la misma moneda, cuya clasificación no me fue fácil en un lote procedente de Cazalla de la Sierra (Sevilla) el año 1985.

Al final descubrí que se trataba de una moneda judía que regalé a través de mi amigo León Levi al museo de Jerusalén (conservo una carta de agradecimiento del profesor Yaakov Meshorer curator de numismática de dicho museo en 1985).

Tanto la hallada en Cazalla de la Sierra como la encontrada recientemente en Melilla, proceden, según Leandro de Villaronga, de Judea.

A esta dedución se llegó, después de un estudio realizado por el Museo Arqueológico de Barcelona (E. Ripoll, J. M" Nuix, L. Villaronga "Monedas de los judíos halladas en las excavaciones de Emporión", Numisma 138-143, 1976. 159-166) de un lote de monedas procedentes de las excavacines de Emporión, entre las cuales aparecieron algunas judías, iguales a las nuestras por la descripción que hacen de ellas.

Se trata de monedas pequeñas (no llegan a los dơs gramos de peso) carentes de valor crematístico, por lo que su presencia en Emporión (ni en Melilla) no obedecía a motivos comerciales ni tenían un fin de atesoramiento. En ellas sólo cabía ver un valor sentimental para sus poseedores los judíos de la diáspora, como un símbolo de su patria lejana, perfectamente explicable, dado el carácter de este pueblo.

La explicación que se da a estas monedas como hipótesis de trabajo, es la existencia en Emporión (lo mismo en Melilla) de una comunidad judía en la primera mitad del siglo I de Cristo, faltando los documentos epigráficos y arqueológicos. "Una vez más corrabora $L$. Villaronga, la numismática sirve de testimonio documental cuando faltan los demás".

En el caso de Melilla, salvo la moneda reseñada, carecemos de documentos históricos que avalen la presencia judía en nuestra ciudad; si bien al ser una ciudad fundada por fenicios con el nombre de Rusadir presumimos la presencia de judíos acompañando a los comerciantes fenicios, algo que solía ocurrir desde los tiempos del rey Salomón (David Rouach Bijoux "Bereberes du Maroc", París 1989, pág. 10). Al parecer los comerciantes hebreos venían a comprar oro a Sala (cerca del actual Sale) en navíos feni- 
cios. Otra ruta del oro y esclavos en tiempos antiguos era la que procedente del Sudán, con escala en Sigilmasa (en el Tafilalet) a través del Muluya alcanzaba los puertos mediterráneos, entre los que sin duda se encontraría Russadir, a quien Plinio le concede el honroso título de Promontorium (ciudad fortificada) y Portum (un puerto del que muchas ciudades costeras carecían). Sobre la presencia de judíos en el Magreb según el rabino Moshe Yacob en su libro Ner Hamariv (Historia de los judíos en el Magreb) constata la existencia de un gran reino constituido en la región del Sous, cuya capital era Oufrane. Este mito de un reino judío, al parecer está vivo hoy día, pues muchos bereberes se creen descendientes de los judíos de aquel tiempo.

Volviendo a nuestra moneda, hallazgo melillense, es igual a otras aparecidas últimamente en Andalucía (desconocemos la existencia de posibles apariciones en el Magreb). Tiene las siguientes características: anverso: racimo de uvas con dos hojas y zarcillos. (la nuestra por estar muy gastada carece de la leyenda que llevan otras del mismo estilo: Loulia), gráfila de puntos y descentrada. Reverso: ánfora con dos grandes asas y las letras L a la izquierda y $\mathrm{D}$ a la derecha del campo.

Parece tratarse de una "Lamed" fenicia y una "delta" griega.

Nuestra moneda hallada en Melilla corresponde a una emisión del año 17 de Cristo de tiempos del emperador Tiberio, siendo procurador romano de Judea (lugar de acuñación) Valerio Grato (15-26 p. C.).

Aunque la moneda acuñada antes del gran Gueruch o Diáspora del año 70, cabría la posibilidad que la trajera a nuestra ciudad algunos de los emigrantes o exiliados de Jerusalén en dicha fecha.

Confiamos en la aparición de nuevas monedas de este tipo, si bien su aspecto poco llamativo pasan desapercibidas para los no expertos.

MONEDAS DE TRAJANO

Aparte la moneda judía, cuyo hallazgo en Melilla no hay duda, adquirí dos monedas más, supuestamente encontradas en Melilla, cerca del verte- 
dero, y próximo al lugar donde se produjo el hallazgo del As Uncial desenterrado al efectuar un hoyo para plantar un árbol.

Este no fue el caso de las dos monedas que paso a describir.

TRAJANO 98-117 p. C.:

$\mathbf{1}^{\circ}$. Sextercio Bronce B.C. Anverso cabeza de Trajano a derecha, IMP. CAES. NERVA TRAIN AUG GERM. P.M. Reverso: figura femenina sentada a izquierda (la Paz). Leyendas frustras que bien podían ser Tr. P. COS II PP. y el exergo S.C. Peso $25 \varnothing$ gramos $34 \mathrm{~mm}$. $\downarrow$ cuño invertido. Curiosamente esta moneda parece coincidir con otra que se encontraba en el museo de la ciudad y que fue sustraida (Trápana $n^{\circ} 2,1988$. pág. 76).

$2^{\circ}$. As bronce B.C. Averso: cabeza de trajano a derecha (la misma leyenda que el sextercio) IMP CAES NERVA TRAIAV AUG GERM P.M. Rverso: TR. POT. COS. III PP. S.C. La figura de la libertad avanzando con escudo. Peso: 8,35 g. Ø $25 \mathrm{~mm}$. $\downarrow$ cuño invertido.

Aparte de estas dos monedas y las que figuraban en el museo municiàl desaparecidas desconocemos la existencia de otras, si bien E. Gonzálvez (op. cit. pág. 119) afirma que "ya hemos señalado en otras ocasiones que el hallazgo de monedas romanas en Melilla es relativamente numeroso y cita a Fernandez de Castro (Aldaba n" 9, 1987, pág. 135). Este cronista de la ciudad contaba la anécdota siguiente: en las inmediaciones de Melilla (Mar Chica) arrojó el mar en distintas ocasiones, según referencias que facilitan los indígenas unos pucheros y ollas de barro cocido, conteniendo pequeñas monedas de cobre, pertenecientes en su mayoría a las legiones romas de Africa, piezas que se hallan en excelente estado de conservación. algunas de las cuales nos fueron cedidas galantemente por el Ayudante de Obras Militares D. Julio Pieri que, residente muchos años en esta ciudad, sostenía frecuente trato con los moros fronterizos". Una verdadera desgracia que estos lotes y tesoros de monedas en "excelente estado de conservación" y por ende fácilmente catalogables no hayan podido ser conocidos por la comunidad científica y su destino nos sea desconocido. No obstante sabemos que el hijo del citado cronista de la Ciudad conserva calcos realizados de las dichas monedas y esperamos nos los facilite para su estudio. 
Una vez más, como diría Leandro de Villarronga, a falta de documentos históricos literarios, la historia recurre a la numismática. Tal es el caso de Melilla. Al carecer de referencias de textos que nos hablen de la ciudad en el siglo III, un importante hallazgo de 35 monedas (en su totalidad sextercios) en un paraje cercano a Melilla, constituye un documento decisivo en orden a elaborar una hipótesis de trabajo sobre la situación de la ciudad en el período indicado.

Las circunstancias del hallazgo de forma casual al ser retiradas las lajas o losas que lo cubrían desde una época tan remota nos induce a pensar que fueron ocultadas deliberadamente ante un peligro inminente.

La zona perteneció a la demarcación de la antigua Mauritania Tingitana y recientemente al protectorado español de Marruecos, concretamente en Annual de trágicos recuerdos para los españoles, donde tuvo lugar uno de los desastres más expectaculares de la historia reciente.

La importancia y trascendencia del hallazgo es triple: en primer lugar por tratarse de un lote y no de monedas dispersas, segundo encontrado en un paraje donde la presencia romana estaba poco documentada y discutida en las fuentes literarias, y por último, actualmente constituye el único o al menos el más importante lote de monedas romanas descubierto en esta zona del Magreb aparentemente poco romanizado. F. Matteu y Llopis (Monedas de Mauritania, Madrid 1949) nos documenta sobre las monedas romanas encontradas en Tamuda (Tetuán) en las excavaciones efectuadas por Tarradell y otras en Alcazarseguer y Taberna.

En dicho lote de 35 monedas (dos frustras) están representados ocho emperadores y una emperatriz: once sextercios pertenecen a Alejandro Severo (221-235 p. C.) dos a su madre Julia Mamea, cinco a Maximino el Tracio (235-238 p. C.), dos a Filipo I el Arabe (244-249), uno a Filipo II (247-249 p. C.), dos a Trajano Decio (249-251 p. C.), uno a Treboniano Galo (251-253) y el último sextercios corresponde a Volusano (251-253 p. C.).

Aún faltando monedas de emperadores que reinaron esporádicamente en este período de crisis, los 35 sextercios cubren todo el período cronoló- 
gico que discurre desde el año 221 al 253 p. G. De los ocho emperados; cinco pertenecen a la dinastía de los Severos y tres a la dinastía de los llamados Ilirios. Se da la circustancia de que el número de sextercios atribuidos a cada emperador está en proporción directa a los años de su reinado como podemos comprobar: Alejandro Severo con 11 sextercios, Gordiano con ocho y Maximino con cinco son los más representados.

Por las fechas indicadas comprobamos que el lote de monedas pertenece al período de la historia romana conocido como la "Crisis del siglo III p. C." y que a juicio de los expertos en este evento, dio comienzo a la muerte de Alejandro Severo el año 238 p. C.

Este lote nos permite, en primer lugar, confirmar noticias ya conocidad de la historia romana, como son el protagonismo que Africa adquiere a raiz de la subida al trono del africano Septimio Severo a finales del siglo $2^{\circ}$ (año 193 p.C). Africa releva y reemplaza a Hispania en la influencia que ésta última gozaba en Roma durante el siglo $2^{\circ}$ a partir de Trajanos y Adrianos.

$2^{\circ}$. En este período se perfila una clara orientación de las relaciones de Hispania hacia Africa preferentemente. J.Mª Blázquez ve con razón esta vinculación con Africa en el desarrollo del comercio africano; la apertura de mercados nuevos en Africa podía compensar la evasión de capitales que Hispania había padecido y aún seguía padeciendo por la obligación de adquirir tierras en Italia, impuesta por los antoninos. (J. Ma Blázquez "La crisis del siglo II en Hispania y Mauritania Tingitana". Hispania XVIII 1968" J. Ma Blázquez "Hispanien unter Antoninen und Severern. Berlín 1975).

En qué grado se llevó esta política económica de acercamiento a Africa. lo ignoramos. En el terreno religioso se constata una clara vinculación con Africa del naciente cristianismo hispano. Esta relación preferentemente de Africa con Hispania culminará en las divisiones de Diocleciano al crearse la "Nova Provincia Hispania Ulterior Tingitana. No olvidemos que las relaciones de las otras provincias africanas, se venían haciendo a través de la Bética. La presencia de tropas hispanas (romanas por redundancia) en Africa, según Blázquez, fueron numerosas si tenemos en cuenta testimonios epigráficos de los dos primeros siglos, en alas y cohortes como el Ala 
III Asturum, la Gohors I Asturum el Gallaecorum, Cohors II Hispana Vasconum, G.R., Cohors II Hispanorum C.R., Cohors I Lema Vorum C.H.

Así pues la epigrafía constata sin duda la presencia en Africa en el siglo II de tropas auxiliares procedentes de Asturias, Galicia y de los Vascones. Estas tropas no sólo permanecieron durante el siglo II p. C. sino que aumentaron en el III al compás del auge que fueron tomando las relaciones Hispania Africa en este período. Así J.M. Blázquez (Historia de España Antigua. Cátedra 1985, pág. 486) constata que durante los Severos la presencia de hispanos en Africa fue muy numerosa.

Es significativa una inscripción del Noricum, referida a un tal "Titus Varius Clemens, Prefectus Auxilia Riorum in Mauritaniam Tingitanam ex Hispania Missorum": se constata el envío de un hispano, Tito Clemente como prefecto de tropas auxiliares a la Mauritania Tingitana.

$3^{0}$. Nos conta que el siglo III marca el máximo de romanización de la Mauritania Tingitana. No somos ajenos a la controversia suscitada entre los expertos sobre la romanización de Africa. Hay historiadores que tratan de demostrar que Roma fracasó en su tentativa de asimilación de los africanos: según ellos éstos rechazaron frontalmente a Roma y su latinidad: Gabriel Camsp. (op.cit. Bereberes 1980, pág. 170) trata de distinguir dos Africas: la del Este (Túnez y Argelias actuales) que fue profundamente romanizada y la del Oeste (actual Marruecos) donde la implantación romana fue más débil. En cualquier carta o mapa del siglo III p. C. cuando la implantación de Roma en Africa adquirió su máximo desarrollo, nos es dado ver esta contraposición. Las principales villas, caminos y calzadas romanas van disminuyendo desde el este, las Syrtes tunecinas hasta el oeste argelino. Sabemos que el puerto más occidental fue colocado durante los severos en el siglo III p. C. no lejos del Muluya cuyo nombre era Numerus Syrorum. Ahí terminaba la calzada romana y comenzaba el desierto que lógicamente se extendía hasta Melilla.

Durante la dominación de Roma en Africa y en especial durante el siglo III p. G., época de máxima expansión, Rusaddir constituyó el auténtico 
cordón umbilical, el único lazo de unión entre las dos Mauritanias, creación de Augusto, las dos Africas ante la carencia de calzadas y corredores terrestres interiores. A pesar de los esfuerzos por localizar una ruta interior como algunos aventuraban, a través del corredor de Taza, el intento hasta ahora ha fracasado.

Las calzadas romanas de la Mauritania Tingitana que a partir de Tingis por la costa, finalizaban en Salé y por el interior en Volubilis, durante el siglo III alcanzaron su máxima penetración en dirección al Atlas Medio. Más allá de Volubilis hacia el sur solamente se han descubierto unos puestos militares en Tocolosida y en Bou Hellu que servían de apoyo a Volubilis: y a partir de aquí había que recorrer 270 kilómetros en línea recta, a vuelo de pájaro a fin de alcanzar el próximo puesto romano establecido en Numerus Syrorum (la actual Maghnia en Argelia). (Paul-Albert Fevrier "Aproches du Magreb Romain 1989). Entre Volubilis en Marruecos y Tlemecén en Argelia, puntos tan alejados no se han encontrado ni huellas ni restos romanos como tierra sigilata, etc.

Las dificultades que Roma encontró en el control de Africa del Norte, en especial de sus sistemas montañosos. (Atlas-Rif, etc.) tuvo que ver sin duda con los pueblos Bereberes. Apelativo éste con el que los bautizó Ibn Jaldum, aunque en realidad ellos mismos se denominaban Imazhiguem, traducido hombres libres.

Las fronteras ollimes colindantes con el desierto desde Trípoli a Marruecos alcanzaron su máxima expansión durante los emperadores Severos, siglo III y la llegada de los camellos a Africa en estos años conducidos por tropas de origen sirio, fue aprovechada por los rebeldes bereberes, quienes obligados por los romanos a emigrar y desplazarse al desierto sahariano, se transformaron en camelleros, subyugando a los negros de los oasis, convirtiéndose en los dueños del Sahara.

Esta transformación afectó más bien a las kábilas bereberes nómadas. no tanto a las sedentarias de las montañas que permanecieron irreductibles al dominio extranjero e impermeables a cualquier tipo de colonización. Sería muy entrado el siglo $\mathrm{XX}$ durante el protectorado franco-español en Marruecos, cuando los rifeños y bereberes del Atlas 
marroquí, se fueron incorporando a la civilización occidental, proceso que aún continua.

A esta visión general de la época de los Severos, en el siglo III podemos añadir hechos concretos y puntuales que tuvieron lugar durante el reinado de los diferentes emperadores en cuya época se acuñaron el lote de 35 sextercios.

A.LSANDHO SEVERO (222-235 P.)

(ver Sha Vita Alex. Sev XXVIII)

Era éste, según sus biógrafos, un emperador bien intencionado, cuando quiso conceder la supremacía a los senadores. Entre sus medidas se cuentan las que acentúan la separación entre ciudadanos y labradores.

Creó una casta o clase de soldados campesinos a los que pagaba, no en dinero, sino en tierras, estableciéndolos en las fronteras: recibieron el nombre Limitanei por estar asentados en el Limes o Frontera.

Esto supuso un aunmento de los efectivos de las legiones establecidas en Africa, pues se aumentaron los puestos militares colocados en los límites del desierto y en lugares montañeses para controlar los irreductibles bereberes entre los que se contaban los rifeños. Los legionarios de Maguncia, no los africanos, asesinaron a Alejandro Severo y a su madre Julia Mamea en marzo del 235, y nombraron emperador a un jefe de origen tracio conocido por su fuerza bruta, Cayo Julio Vero Maximino, quien tomó el nombre de Maximino el Tracio y en él comienza el período conocido por el nombre de Anarquía Militar.

MAXIMINO EL TRACIO (235-2:38 P. (:.)

Con este emperador de breve reinado comienza una de las épocas más calamitosas y anárquicas de la historia de Roma, hasta tal grado que estuvo a punto de dar al traste con el Imperio de Roma. Después de alguna expedición brillantes contra los germanos, vio como las legiones bajo el método de los "pronunciamientos", elegían a su propio emperador y así al senador Quartino lo eligieron sus propias tropas, para ser asesinado a continuación. 
En el Africa proconsular, la indignación contra las exaciones de Maximino el Tracio fue tan general que estalló una revuelta en el campo y no tardó en llegar a Cartago. Los amotinados obligaron al procónsul de Africa, Marco Antonio Gordiano y al legado su hijo del mismo nombre, a proclamarse emperadores (Gordiano I y Gordiano II) a la vez, gobernando simultáneamente.

En la Numidia, región próxima a Melilla, había un legado de legión, Capeliano, favorable a Maximino el Tracio, el cual derrotó y dio muerte a los Gordianos, los cuales habían gobernado sólo tres semanas (febrero del 238 p. C.). Los soldados de Maximino el Tracio amotinados en Aquileia contra él, le causaron la muerte junto a su hijo Maximino, el 10 de mayo del 238 p. C.

COIIANO III (238-2++ P. (.)

Elegido emperador por los pretorianos de Roma: era el tercero, ya que las legiones habían elegido a sus favoritos Pupieno y Balbino, asesinados estos dos últimos. Las provincias, a falta de candidatos, reconocieron a Gordiano III, quien pudo gobernar un período largo para los tiempos que corrían, seis años.

Tenía sus adictos en la Mauritania cuyo procurador abortó el año 240 la proclamación como emperador, en lugar de Gordiano, del procónsul de Africa, Sabiniano.

Filipo I el Arabe (244-249 p. C.) sucedió a Gordiano, asesinado éste en un motín de la tropa, lejos de Roma y de Mauritania en el valle del Eúfrates. Asoció al trono o su hijo del mismo nombre Filipo II (247-249 p. C.) y ambos gobernaron conjuntamente.

Durante el reinado de los Severos, Africa, jugó un papel importante en los asuntos del Imperio y sobre todo, la Iglesia, cuyos obispos llegaron a ejercer el cargo de "Procuradores Imperiales".

El cristianismo tuvo tal auge en estas tierras del Norte de Africa al punto que según San Jerónimo y el obispo Orosio, el emperador Filipo se convirtió a esta religión oriental y tuvo qe sufrir penitencia impuesta por un obispo a la muerte de Gordiano III. L̇a suerte de la iglesia cambió a la 
llegada de los nuevos emperadores Ilirios que de ser favorecida pasó a ser perseguida.

Decio (249-251 p. C.) derrotó y dio muerte a Filipo I (septiembre del 249 p. C.) cerca de Verona, al tiempo que el hijo de éste Filipo II caía asesinado en Roma en el campamento de los pretorianos.

A su vez Decio con sobrenombre Trajano a quien se le atribuye la primera persecución de la iglesia, fue traicionado y muerto por uno de sus propios generales cuando se encontraba luchando contra los bárbaros godos. Este general se proclamó emperador con el nombre de Treboniano Galo (251-253 p. C.) y asoció al trono como César a su hijo Galo Veldumniano Volusiano (251-253 p. C.), siendo ambos derrotados y muertos en mayo del año 253 por Emiliano.

Se deduce de lo expuesto que la vida de los emperadores que figuran en nuetras sextecias fue muy agitada en consonancia con los tiempos y la muerte a la mayoría de ellos les sobrevino violentamente.

La mayor parte de estos emperadores, en particular los Severos, tuvieron una relación especial con el norte de Africa, ya que contaron con la ayuda, en los momentos críticos, de procuradores y legados de la Numidia y la Mauritania.

Tuvo lugar en Anual (famoso por el desastre que padecieron las tropas españolas el año 1921) perteneciente en la actualidad a la Cábila de Temsamán (beni-Ulichek) a una distancia de $80 \mathrm{kms}$. de Melilla, en zona que perteneció al Protectorado Español hasta 1958, y que en la antigüedad perteneció a la provincia romana de la Mauritania Tingitana anexionada a Roma en el año 54 p. C. en tiempos del emperador Calígula, al ser asesinado su último rey Ptolomeo. Los sextercios fueron acuñados en el siglo III cuando la provincia había sido anexionada dos siglos antes a Roma.

Tratando de rastrear la presencia romana en dicho lugar a través de las fuentes literarias, hemos encontrado dos que podrían darnos alguna luz: el 
conocido "Itinerarium Antonini" y el geógrafo griego Claudio Ptolomeo.

Claudio Ptolomeo, geógrafo del siglo II p. C. menciona diversos topónimos de la costa mediterránea, entre los que se encuentra Russadeiron y le da las coordenadas de $10-34^{\circ} 45^{\prime}$. Los investigadores coinciden en identificar este topónimo con Rusadir, la actual Melilla y al occidente a 10-341 colocan Sextiaria Acra, o cabo Sextiaria, con sólo 45' minutos alejado de Rusadir, si bien este punto parece coincidir con la actual Alhucemas, no se puede descartar localizarlo cercano al actual Annual.

Sin duda el documento más importante es el "Itinerarium Antonini", especie de guía turística confeccionada según unos en época del emperador Caracalla (198-217 p. C.), principios del siglo III y últimamente los investigadores la retrotraen a finales del siglo en tiempos del emperador Diocleciano. En cualquier caso, el documento refleja los años en que fueron acuñados los citados sextercios o poco antes o poco tiempo después.

El itinerario no coincide con los topónimos de Ptolomeo: solamente Tenia Loga y Rusadir son localizados en ambos. El cabo Sestiaria, comentado anteriormente no aparece en el itinerario. En su lugar está "Ad Sex Insulas" que según los comentaristas se refiere lo mismo que el anterior, aAlhucemas, localidad cercana a Anual.

El itinerario no da coordenadas, sino que calcula las distancias a partir de Tingis (Tánger). Entre Sex Insulas (probable Alhucemas) y Rusadir Colonia (Melilla) aparece el nombre "Promontorium Camnarum" a 65 millas al occidente de Rusadir, Melilla. Curiosamente esta distancia de 90 kilómetros es la misma que se da actualmente entre Melilla y Anual.

Enrique Gonzalvez (Ciudad Antigua de Rusadir 1991, pág. 126) escribe sobre el Promontorium Cannarum lo siguiente: "Indudablemente coincide con la "Punta Afrau". Allí existen los restos de una antigua población dependiente estrictamente del mar por no ser posible la práctica de la agricultura en muchos kilómetros a la redonda. Coincido y estoy de acuerdo con el amigo Gonzalvez en la identificación del Promontorium-Cannarum en la Punta Afrau en la actualidad.

Discrepo con la inexistencia de cultivos. Tanto el río Amekran al occidente como el Tazaguin, sobretodo el primero, lleva agua abundante a lo largo 
de casi todo el año, lo que permite cultivos importantes en las hondonadas y planicies intercaladas entre las alineaciones montañosas. El lugar se nos ofrece como un gran circo, rodeado de montañas y abierto al mar a través de una larga playa de varios kilómetros, donde los españoles construyeron dos puertos: Sidi Dris al oeste y Pumta Afrau al este. Punta Afrau está cercana a Anual, a unos $10 \mathrm{kms}$. y en línea recta se acortan los kilómetros.

Gonzalvez cita a Morán Bardón ("Visita arqueológica a Marruecos". Africa, diciembre, 1950) quien señala "la presencia de restos antiguos: entre ellos cerámica pintada, que por sus características es preislámica". También indica este autor "la existencia de trozos de lucernas muy antiguas".

Entre Punta Afrau (Promontorium Cannarum) y Anual existe una mina, explotada durante el protectorado, por los expañoles: en la playa quedan restos del embarcadero del mineral de hierro y había en proyecto una conducción aérea que no llegó a concluirse. Mina, que según el citado Morán Bardón, quien la visitó con anterioridad había sido explotada en época medieval e incluso en la antigüedad romana. "A destacar, dice, que una de las cámaras de grandes dimensiones (150 metros de longitud por 10 metros de ancho y 10 metros de alto) recibe el nombre de cámara del "Elefante de Anibal" siendo probable su explotación en la época romana".

El tesoro de 35 sextercios encontrado en Annual no lejos de las tres colinas donde Manuel Fernández Silvestre acantonó sus tropas: los regimientos de Ceriñola y Regulares y la Columna Africa, tuvo que ser escondido con motivo de algún grave acontecimiento y la fecha de acuñación nos lleva precisamente a los años álgidos de la crisis del siglo III que sufrió el Imperio Romano.

No es descartable que las kábilas que rodeaban el lugar (auténtica ratonera) los ancestros de los actuales Ait Uriaghar y Temsamanis y Beni-Said atacaran al destacamento romano y lo liquidaran, repitiéndose la historia dieciocho siglos más tarde cuando el rifeño Mohamed Ben Abdelkrim el Jatabi atacó al general español Silvestre en el mismo lugar, produciéndose uno de los desastres más espectaculares de nuestra reciente historia. En ambos casos las tropas ocupantes sufrirían un duro castigo por parte de los ocupantes. 
El hallazgo de este tesoro coincide con otros encontrados en Volubilis y nos señalan el abandono del territorio. Las monedas de Volubilis con fechas de emperadores que no se alejan más allá de Numeriano (283-284) nos indican el abandono de esta colonia treinta años más tarde que la nuestra de Annual y nos atrevemos a decir que la caida de la posición de Annual trajo como consecuencia la caida y desaparición de Rusaddir. De esta ciudad no se vuelve a escribir de ella, salvo si se admite la existencia de un obispado en el siglo VII, hasta entrado el siglo $\mathrm{X}$ con cambio de nombre, que pasó a denominarse Malila, la actual Melilla.

\section{RAZONES PARA EL ESTACIONAMIENTO}

Del examen del tesoro compuesto como ya hemos indicado por treinta y cinco sextercio, monedas todas ellas de bronce, llama la atención la ausencia de otro tipo de monedas como podían ser áureos (Paul-Albert Fevrier "Aproches du Maghreb Romain". Aix-En-Provence-1989), y quinarios de oro o como suele ocurrir en otros hallazgos, denarios y quinarios de plata y sobretodo los antoninianos acuñados por primera vez por el emperador Caracalla hacia el 214 p. C.

Esta ausencia de monedas de oro y plata, parece sugerirnos que difícilmente podemos pensar en comerciantes que traficaban en esta región del Norte de Africa y que como sabemos tomó auge importante en estos años en detrimento de Hispania. No podemos menos de pensar en gentes que hacían sus trueques con monedas de bronce, las cuales constituían su único tesoro y escondían ante una situación de peligro. Nos estamos refiriendo a la presencia en Annual de soldados Limitanei.

Estos soldados de frontera o Limitanei, como bien dice E. Gonzalves (op. cit. pág. 139) "Alejandro Severo creó el sistema de los soldados Limitanei, es decir los soldados agricultores. (No olvidemos que una tercera para de los sextercios corresponde a este emperador). Consistía en la donación de tierras fronterizas a soldados con tal que sus herederos se enrolasen en el ejército y con la obligación de defenderlas. "Vita Alex Sev LVIII". 
La única objeción que le puedo hacer a $\mathrm{E}$. Conzalvez es que la presencia de tales tropas las coloca en Rusadir, cuando sabe bien que en las ciudades se daba otro tipo de soldados conocidos por el nombre de Comitanei. Tropas de acción rápida para acudir a cualquier evento que se produjera en torno a la ciudad.

Las tropas Limitanei, durante los Severos quedaron acantonadas en los puertos que limitaban con el desierto o en lugares próximos a las montañas donde habitaban tribus o kábilas bárbaras (en nuestro caso bereberes).

Ni Volubilis ni Rusadir al decir del citado autor elevadas a la categoría de colonias podían tener Limitanei, más bien Comitatensis.

La región de Anual que no forma una hoya propiamente dicha como las que existen al otro lado del mar de Alborán, Málaga, Vélez Málaga. Almuñécar, Adra, etc. sino que aparece como un gran circo entre Cabo Quilates al oeste y Punta Afrau al este, formado por la erosión de sus pequeñas estribaciones montañosas y que forman pequeños acantilados al llegar a la gran playa abierta al mar Mediterráneo. $\mathrm{Al}$ oeste y separada por los montes de Abarran (576 metros), Kan (1129 metros) y Bumesaud (1231 metros) está la Bahía de Alhucemas, verdadera hoya ocupada por el mar y colmatada por los derrubios que portan los ríos Nekor y Ghis. Alhucemas la identificamos en el Cabo Sextaria de Claudio Ptolomeo (siglo II p. C.) y las "Sex Insulae" del Itinerario de Antonino.

Tanto en la Bahía de Alhucemas como en la depresión de Anual se dan condiciones óptimas para el acantonamiento de tropas Limitanei o fronterizas. En torno a Alhucemas pululan feroces cábilas que han dejado sentir su agresividad durante las guerras del Rif (1921-1927): Ait-Uariaghar-Gueznaias-Ait Bocoias por citar las más importantes.

En torno a Anual, han venido habitando sus montañas los ya nombrados Ait Uariaghar (Beni-Urriaguel para los españoles) Temsamanis (fracción de los Ait-Ulichek), los Ben Said, Beni-Tuzin y otras cábilas menores. Todas las nombradas más los M:Talsas y Beni-Bu-Yahin infrigieron un duro correctivo al ejército español. Estimamos que su ferocidad no fue menor en los años del Imperio Romano. 
Estas tropas Limitanei tenían como principal cometido el control de las ya citadas cábilas, impidiendo con ello que se lanzaran sobre los dos enclabes romanos más importantes: el de la colonia Rusadir en el litoral mediterráneo junto a Tres Forcas y la Colonia Volubilis al interior en un lugar rico en cereales para abastecer Roma y su ejército.

Estos dos puntos tuvieron que ser objetivos preferentes de defensa en la estrategia de Roma. Rusadir por el el único punto de enlace, como puerto, entre la antigua Cesariense y la Tingitana, y Volubilis, granero del Imperio.

El hallazgo de los sextercios en Anual podría significar la pérdida de los puertos que defendían a Rusadir. En toda la costa desde Tamuda (Tetuán) a Tres Formcas el tandem Alhucemas-Anual ofrecen los puntos más estratégicos para esta defensa. Las tropas allí estacionadas tenían las suficientes tierras de labor y recursos pesqueros para poder subsistir (economía de subsistencia) sin necesidad de ser abastecidas.

La caida de Anual, recalcamos, supuso la inevitable caida de Rusadir. Los esfuerzos beneméritos que hace Gonzalves en su historia de la ciudad de Rusadir por hacerla sobrevivir a la Crisis del siglo III no se fundamentan en argumentos de mediana solidez, según él mismo se ve obligado a admitir.

También la caida de Anual arrastró la caida de Volubilis. Los Baquatas cábilas rifeñas emparentadas con las nuestras la atacaron y obligaron a pactar con ellas al emperador Probo (276-258 p. $\mathrm{C}_{3}$ ) confirman el abandono de dicha colonia. Al argumento de los tesoros escondidos se añaden las últimas dedicaciones a emperadores en inscripciones de altares (la última el 276 p. C.) y por último las excavaciones arqueológicas.

Nosostros solamente contamos con el tesoro de monedas a falta del hallazgo de lápidas, inscripciones y sobretodo de excavaciones.

Para abundar en el paralelismo entre Volubilis y Rusadir diremos que una de las inscripciones cristianas del Magreb datable del año 655 p. C. se ha encontrado en Volubilis confirmando cierto renacimiento de la ciudad después de haber desaparecido como colonia romana. Rusadir parece ser, tuvo un renacimiento parecido, al gozar de un obispado en dicho siglo, 
principios del VII, si nos atenemos al controvertido documento conocido por el nombre de Thronus Alexandrinus.

Para terminar citamos a Leandro Villarronga "afirmando que a falta de documentos históricos, epigráficos y arqueológicos, la numismática aporta un testimonio documental".

Este es nuestro caso y por ello la importancia que hemos atribuido al hallazgo y tenemos la confianza y la esperanza de que se produzcan otros. 